\title{
Universal Conductance and Conductivity at Critical Points in Integer Quantum Hall Systems
}

\author{
L. Schweitzer ${ }^{1}$ and P. Markoš ${ }^{2}$ \\ ${ }^{1}$ Physikalisch-Technische Bundesanstalt, Bundesallee 100, 38116 Braunschweig, Germany \\ ${ }^{2}$ Institute of Physics, Slovak Academy of Sciences, 84511 Bratislava, Slovakia
}

(Dated: October 29, 2018)

\begin{abstract}
The sample averaged longitudinal two-terminal conductance and the respective Kubo-conductivity are calculated at quantum critical points in the integer quantum Hall regime. In the limit of large system size, both transport quantities are found to be the same within numerical uncertainty in the lowest Landau band, $0.60 \pm 0.02 e^{2} / h$ and $0.58 \pm 0.03 e^{2} / h$, respectively. In the 2 nd lowest Landau band, a critical conductance $0.61 \pm 0.03 e^{2} / h$ is obtained which indeed supports the notion of universality. However, these numbers are significantly at variance with the hitherto commonly believed value $1 / 2 e^{2} / h$. We argue that this difference is due to the multifractal structure of critical wavefunctions, a property that should generically show up in the conductance at quantum critical points.

PACS numbers: 72.15.Rn, 71.30.+h
\end{abstract}

The transitions between quantized Hall plateaus are generally viewed as a manifestation of continuous $T=0$ quantum phase transitions. They emerge in the presence of a strong magnetic field in disordered two-dimensional electron or hole gases which can be observed in Silicon MOSFETs and GaAsheterostructures. According to renormalization group considerations, some properties of the quantum phase transitions should be universal, i.e., independent of microscopic details of the system. For instance, this is supposed for the critical exponent that controls the divergence of the localization length $\xi(E)=\xi_{0}\left|E-E_{c}\right|^{-\mu}$ near the critical energies $E_{c}$, a behavior which has been corroborated by experimental [1, 2] and numerical [3, 4, 5] studies. In addition, it has been suggested that the impurity averaged elements of the conductivity tensor act as coupling constants in an appropriate field theory [6] where all the transitions belong to the same universality class [7] with universal values of the critical dissipative and Hall conductivities. It has been proposed [8, 9] that their values $\sigma_{x y}^{c}=(n-1 / 2) e^{2} / h$ and $\sigma_{x x}^{c}=1 / 2 e^{2} / h$ are simply related at the respective $n$-th transition.

Away from the critical points, the Hall conductivity is exactly quantized due to topological reasons. Thus, in the presence of electron-hole symmetry, the above formula for $\sigma_{x y}$ is evident at the transition points [10]. Support for the existence of a universal $\sigma_{x x}$ comes both from experiments [11, 12] and numerical calculations. Amongst the latter, a critical conductivity $\sigma_{x x}^{c}=0.54 e^{2} / h$ was found for the lowest Landau band using white noise disorder potentials [13]. A similar diagonalization study [10] yielded $\sigma_{x x}^{c}=(0.55 \pm 0.05) e^{2} / h$ for various correlated random potentials, a result considered by the authors to be consistent with the prediction [8] $\sigma_{x x}^{c}=1 / 2 e^{2} / h$. However, the critical diagonal conductivity deduced from a Thouless number study [14] exhibited a strong dependence on the range of the scatterers. Moreover, calculations of the two-terminal conductance of square systems produced a significantly larger value $g_{c}=(0.58 \pm 0.03) e^{2} / h[15]$ and showed considerable fluctuations exhibiting a broad distribution between zero and $e^{2} / h$ [16]. Here $g_{c}=\lim _{L \rightarrow \infty} \sum_{i} g_{i}^{c}(L) / N$ is the disorder aver- aged critical conductance of $N$ realizations. This value was derived from a finite-size scaling study of the transfer-matrix results calculated within a Chalker-Coddington (CC) network model [3]. In a recent work, this outcome has been confirmed with $g_{c}=(0.57 \pm 0.02) e^{2} / h$ 17].

On the other hand, similar transfer-matrix calculations within a tight-binding (TB) lattice model with spatially uncorrelated disorder potentials yielded $g_{c}=(0.506 \pm 0.01) e^{2} / h$ [18], hence, the asserted universality cannot be taken for granted. Even if it were possible to reconcile the contradicting results, the hitherto unknown relation between the twoterminal conductance and the conductivity, the latter calculated for instance from the Kubo formula for systems without contacts and leads, would still inhibit us drawing final conclusions about the validity of the proposed universal value of the critical dissipative conductivity $\sigma_{x x}^{c}=1 / 2 e^{2} / h[8]$.

Our work is aimed at resolving this issue by making available the missing links and therewith provide evidence for an equality of conductivity and conductance at the critical point in the lowest Landau band. We find, within the uncertainty of our calculations, similar values $0.58 e^{2} / h$ and $0.6 e^{2} / h$, respectively. These values are, however, significantly larger than the previously proposed and well accepted $\sigma_{x x}^{c}=1 / 2 e^{2} / h$. The origin of this discrepancy is suggested to arise from the multifractal correlations of the critical eigenstate amplitudes, which were presumably not fully taken into account previously. We also present the critical conductance distribution for the second Landau band which agrees with the one from the lowest. The scaling of the ensemble averaged $g_{c}$ yields $(0.61 \pm 0.03) e^{2} / h$, a result that supplies further evidence for the existence of a universal conductance.

We first show that for non-interacting electrons moving in a quenched disorder potential and perpendicular magnetic field described by a tight-binding lattice model the following relation holds,

$$
g_{c}(L)=g_{c}(\infty)-g_{0}\left(L_{0} / L\right)^{y},
$$

if the size of the square systems is $L>L_{0}$. We find in the lowest Landau band a critical conductance $g_{c}(\infty)=(0.60 \pm$ 
$0.02) e^{2} / h$ with $y=0.4 \pm 0.02$. Such a relation has been suggested previously [19] where the least irrelevant scaling exponent $y$ was proposed to equal the exponent of the critical eigenfunction correlations [13]. Then, we demonstrate that the Kubo-conductivity for very long strips of width $M>M_{0}$ obeys a similar power law

$$
\sigma_{x x}^{c}(M)=\sigma_{x x}^{c}(\infty)-\sigma_{0}\left(M_{0} / M\right)^{y_{1}}
$$

with $\sigma_{x x}^{c}(\infty)=(0.58 \pm 0.02) e^{2} / h$. The irrelevant scaling exponent $y_{1}$ is $0.38 \pm 0.03$. Our value of the critical conductivity is in good agreement with the two-terminal conductance, but is significantly larger than predicted previously [8].

The movement of non-interacting charge carriers in a disordered two-dimensional system in the presence of a perpendicular magnetic field $B=\phi \hbar /\left(e a^{2}\right)$ is described by a tightbinding model on a square lattice with lattice constant $a$, and $\phi /(2 \pi)$ is the number of flux quanta per plaquette,

$$
\begin{aligned}
\mathcal{H} / V & =\sum_{x y} w_{x y} c_{x y}^{\dagger} c_{x y}+c_{x y+a}^{\dagger} c_{x y}+c_{x y-a}^{\dagger} c_{x y} \\
& +\sum_{x y} \exp (i \phi y) c_{x+a y}^{\dagger} c_{x y}+\exp (-i \phi y) c_{x-a y}^{\dagger} c_{x y}
\end{aligned}
$$

The distribution of spatially Gaussian correlated on-site disorder potentials $w_{x y}$ with zero mean has been generated as described in Ref. 20 from uncorrelated random numbers evenly distributed between $[-W / V, W / V]$. Here, $W$ is the disorder strength in units of the transfer term $V$ and $\left\langle w_{x y} w_{x^{\prime} y^{\prime}}\right\rangle \sim$ $\exp \left(-\left[\left(x-x^{\prime}\right)^{2}+\left(y-y^{\prime}\right)^{2}\right] /\left(2 \kappa^{2}\right)\right)$ defines the potential's correlation length $l_{p}=\sqrt{2} \kappa$ which was varied in the range $0<\kappa \lesssim l_{B}$, where $l_{B}=a / \sqrt{\phi}$ is the magnetic length.

In contrast to the one-band model [10, 13] or the network model [15, 17], the energetical position of the transition points are usually not known a priori in the TB model. They depend on disorder strength, correlation length, and magnetic field. Therefore, careful extensive calculations had to be carried out to precisely detect the critical points. A slight imprecision influences considerably the results for the conductance and the conductivity. We used various methods like calculations of the energy and size dependence of level statistics [20], localization length [21], and Hall conductivity to find the exact critical energy. The first quantity was obtained by direct diagonalization whereas the latter two were calculated by means of a recursive Green function technique [22]. The two-terminal conductance of a $L \times L$ sample with two semi-infinite ideal leads attached to opposite sides and periodic boundary conditions applied in the transverse direction was calculated via the Landauer formula $g=\operatorname{Tr} t^{\dagger} t$ where $t$ is the transmission matrix. For a given disorder realization the conductance was numerically evaluated using a well known algorithm [23]. Ensembles of at least $N=10^{5}$ realizations were used $\left(N>10^{4}\right.$ in case of $L / a=512$ ) to determine the conductance distribution and the corresponding mean critical conductance $g_{c}(L)$. The critical conductivity was obtained within linear response theory using a recursive Green function method [22].

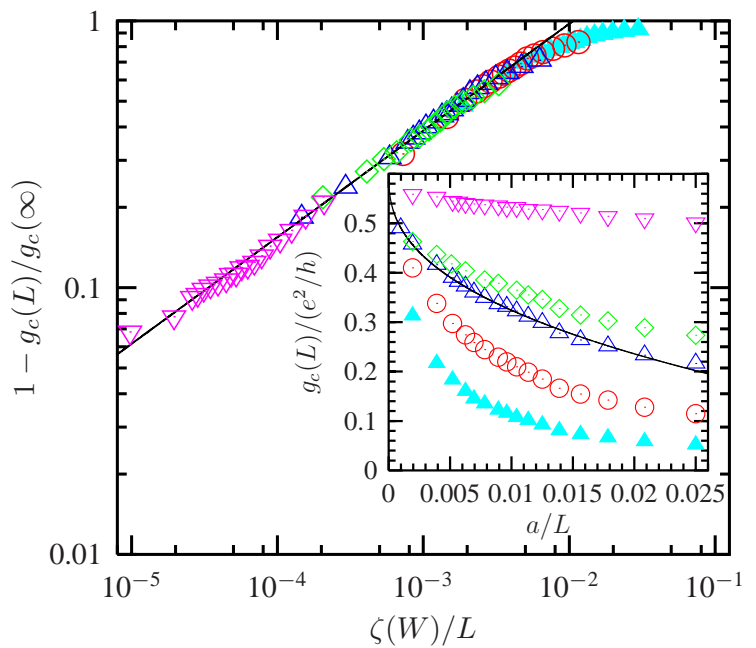

FIG. 1: (color online). The relative deviation of the mean critical conductance $g_{c}(L)$ from $g_{c}(\infty)$ vs. rescaled inverse system size $L$ for a disorder potential with correlation $\kappa / a=1.0$, disorder strengths $W / V=0.125(\mathbf{\Lambda}), 0.25(\bigcirc), 0.5(\triangle), 0.7(\diamond), 6.0(\nabla)$, and $B=1 /\left(8 a^{2}\right) \cdot h / e$. The best fit gives $g_{c}(\infty)=0.6 \pm 0.02$ and a slope $y=0.4$. The inset shows the unscaled data for $L / a>40$.

The mean critical conductance $g_{c}$ depends on the system's size $L$, the disorder strength $W$, and the correlation length $l_{p}=\sqrt{2} \kappa$ of the spatially correlated disorder potentials. In order to detect the genuine size dependence and to be able to correlate our results with those obtained in the presence of only one Landau band [10, 13, 15, 17], one has to confine the parameter range to the situation where the width of the disorder broadened Landau bands $\Gamma(W, \kappa)$ is small compared to their energy separation $\hbar \omega_{c}$. Stronger disorder enhances those matrix elements that couple neighboring Landau bands giving rise to an increasing $g_{c}$ due to extra scattering events into the next band, and eventually, shifts the critical states to higher energy until they disappear. This levitation of the current carrying states was shown to depend essentially on the correlation length [21]. The size dependence of the mean conductance was calculated for various disorder strengths $W$ and correlation parameters $\kappa / a=0.3,1.0$, and 1.5. For disorder potentials with $\kappa / a=1.0$, which corresponds to a correlation length of the order of the magnetic length $\left(l_{p} / l_{B}=1.2533\right)$, the rescaled mean conductances are shown in Fig. 1 for system sizes $24 \leq L / a \leq 512$ and disorder strength $0.125 \leq W / V \leq 0.7$ and $W / V=6.0$. In addition, the average over 1610 realizations for systems of size $L=1024$ and $W=0.5$ is also included. The inset shows the original data for $L \geq 40 \mathrm{a}$. The best fit, leading to a mean critical conductance $g_{c}(\infty)=(0.60 \pm 0.02) e^{2} / h$ and an irrelevant scaling exponent $y=0.4 \pm 0.02$ is also shown in the inset of Fig. 1] (solid line). Our mean critical conductance is in accordance with the results reported for the network model [15, 17].

The rescaling of the data according to $g_{c}(\infty)-g_{c}(L) \propto$ $(\zeta(W) / L)^{y}$ is in principle possible only for small disorder 


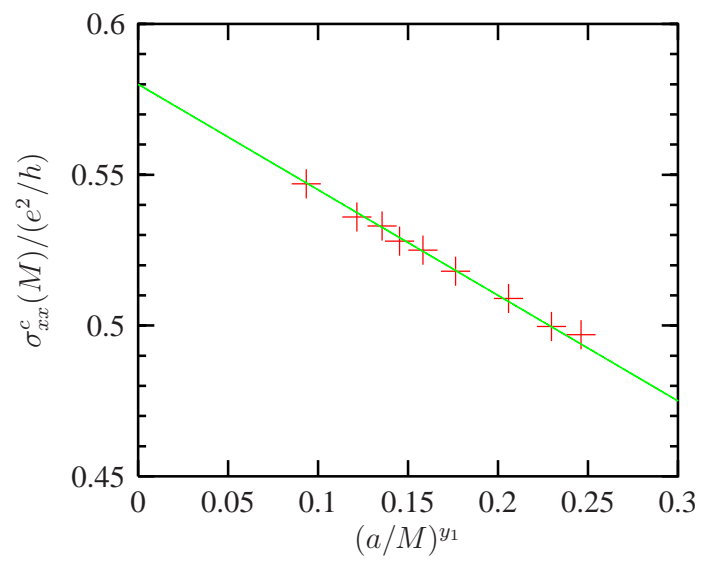

FIG. 2: (color online). The critical longitudinal conductivity vs. inverse system width $M$ for a correlated disorder potential with $W=0.5, \kappa / a=0.3$, and magnetic flux density $B=1 /\left(8 a^{2}\right) \cdot h / e$. The fit to Eq. 2] gives $\sigma_{x x}^{c}(\infty)=(0.58 \pm 0.02) e^{2} / h$ and $y_{1}=$ $0.38 \pm 0.03$.

strength. For stronger disorder $(1.0 \leq W / V \leq 5.0)$, the true scaling regime cannot be reached for system sizes $L / a<192$ because Landau band mixing, which is obvious from the strong overlap seen in the density of states $\varrho$, causes scattering into the critical states in higher Landau bands. This disorder induced Landau band coupling may be the reason for the differing result $g_{c}=(0.506 \pm 0.01) e^{2} / h$ of Ref. 18. Increasing the disorder strength further, one reaches the situation where critical states in higher Landau bands get annihilated by their corresponding anti-Chern states [20]. For $W / V=6.0$, when only the critical state of the lowest Landau band survives, scattering into higher conducting states is not possible so that the conductance data again show scaling and match up with those obtained for small disorder. A similar behavior is observed for weak correlated disorder potentials with $\kappa / a=0.3$, where $l_{p}=0.424 a$ is less than half the magnetic length. In this case, we found $g_{c}(\infty)=0.59 \pm 0.03$ and $y=0.42 \pm 0.03$ from the rescaled data (not shown).

Having established the critical conductance in the lowest Landau band, we turn now to our results obtained from the investigation of the critical Kubo conductivity for disorder potentials with $\kappa / a=0.3$. In Fig. 2 2 the dependence of $\sigma_{x x}^{c}$ on the width of the system is shown in the range $40 \leq M / a \leq$ 512. We find in the large size limit $\sigma_{x x}^{c}=(0.58 \pm 0.03) e^{2} / \bar{h}$ which is significantly larger than proposed previously.

The reason for a critical conductance larger than $0.5 e^{2} / h$ and, therefore, for the discrepancy with the suggested behavior involving quantum percolation at the critical point in the integer QHE [8, 9, 24] may be associated with the multifractality of the critical quantum Hall eigenstates [25, 26, 27, 28, 29], a property that was not fully appreciated at that time. The diffusion coefficient $D$, determining the conductivity $\sigma_{x x}=e^{2} \varrho D$, is essentially influenced by the multifractal correlations of the wave function amplitudes [13, 29, 30, 31]. Fal'ko and Efetov have shown [32, 33] that for length scales

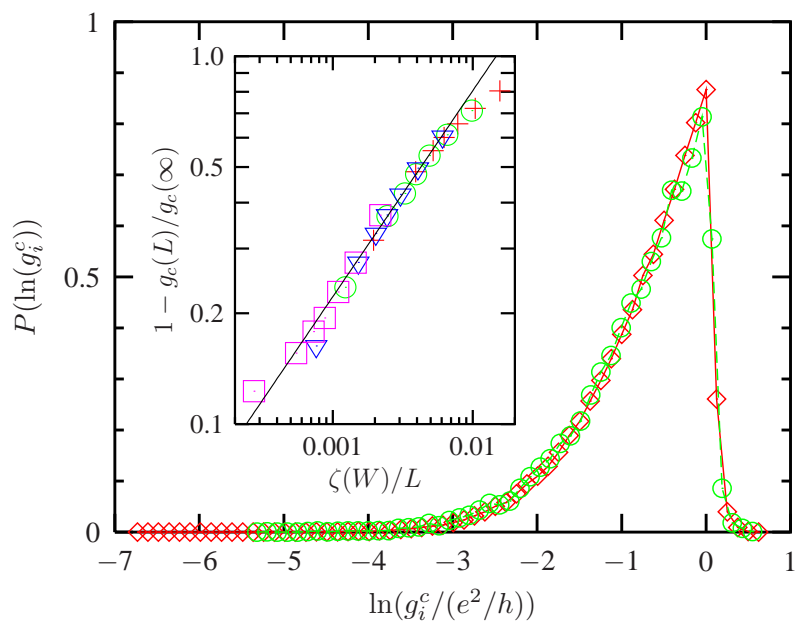

FIG. 3: (color online). The critical conductance distribution functions $P\left(\ln \left(g_{i}^{c}\right)\right)$ from the lowest $(\diamond, L / a=192, W / V=$ $\left.6.0, \kappa / a=1.0, B=1 /\left(8 a^{2}\right) \cdot h / e\right)$ and the second lowest $(\mathrm{O}$, $\left.L / a=512, W / V=0.5, \kappa / a=1.5, B=1 /\left(32 a^{2}\right) \cdot h / e\right)$ Landau band (LB). The inset shows the relative deviation of the mean critical conductance $g_{c}(L)$ for the 2 nd LB vs. rescaled inverse system size $L$ for a disorder potential with correlation parameter $\kappa / a=1.5$ and disorder strengths $W / V=0.125(+), 0.18(\bigcirc), 0.25(\nabla)$, and 0.5 ( $\square)$. The best fit gives $g_{c}(\infty)=(0.61 \pm 0.03) e^{2} / h$ and a slope $y=0.56 \pm 0.05$

shorter that the localization length, these correlations are also present away from the Anderson transition. Using a special version of the supersymmetric $\sigma$ model, they provided a formula relating the diffusion coefficient to the generalized multifractal dimensions $d(q)$,

$$
d(q)=2-\frac{q}{4 \pi^{2} \varrho D \hbar} .
$$

This relation for unitary symmetry was obtained in the leading order in $1 /(2 \pi \varrho D \hbar)$ and should hold for $q \leq 2 \pi \varrho D \hbar$. We assume Eq. (4) to be exact near the quantum Hall critical point for electronic states with localization length larger than the system size [33]. Taking $q=1$, we find very good agreement with our numerical results presented above,

$$
\sigma_{x x}=\frac{1}{2 \pi(2-d(1))} \frac{e^{2}}{h}=0.61 \frac{e^{2}}{h},
$$

using the information dimension $d(1)=\alpha(1)=1.739 \pm$ 0.002 derived [34] from the precise parabolic $f(\alpha(q))$ singularity strength distribution. Over the years, convincing numerical evidence for a universal $f(\alpha(q))=2-(\alpha(q)-$ $\left.\alpha_{0}\right)^{2} /\left[4\left(\alpha_{0}-2\right)\right]$ has been accumulated for different quantum Hall models [27, 28, 34, 35, 36]. Within error bars, the whole distribution appears to be independent of disorder strength, potential correlation length, and magnetic field. It is determined only by a single value $\alpha_{0}=2.262 \pm 0.003$ [34]. Therefore, also $d(1)$ must be universal and so should the critical conductivity, if Eq. [5] was exact.

We also found the multifractal properties of the wavefunctions to be independent of the Landau band index for cor- 
related disorder in agreement with previous investigations [37]. Therefore, to check the universality of Eq. [5, we calculated the critical conductance in the 2nd Landau band within the same model, but for weaker magnetic field $B=$ $1 /\left(32 a^{2}\right) \cdot h / e$ and stronger disorder correlations $\kappa / a=1.5$ $\left(l_{p} / l_{B}=0.94\right)$. We found $g_{c}(\infty)=(0.61 \pm 0.03) e^{2} / h$ (see inset of Fig. 3 from the scaling of the mean critical twoterminal conductance, which is in good agreement with our results for the lowest Landau band. The critical conductance distribution functions $P\left(g_{i}^{c}(L)\right)$ for the 1st and 2nd Landau band of our TB model (shown in Fig. 3) are almost identical and look the same as the one reported for the CC model [38]. Conductance values larger that $e^{2} / h$ reflect the fact that more than one transmission channels are effective, due to the twodimensional contacts. This is contrary to investigations of the critical point-contact conductance [39, 40] which show a flat symmetrical distribution between zero and $e^{2} / h$ and, therefore, a mean conductance $g_{c}=0.5 e^{2} / h$.

A universal critical conductivity $\sigma_{x x}^{c} \simeq 0.6 e^{2} / h$ should be easily observable in experiments on Corbino disks in the low temperature limit. Here, the disagreement with the value $1 / 2 e^{2} / h$ should be clearly discernible and, for the first time, reveal the multifractality of the critical eigenstates in a transport measurement. In case of the usual Hall bars, using our new value for the critical conductivity, the critical resistance $\rho_{x x}^{c}=\sigma_{x x}^{c} /\left[\left(\sigma_{x x}^{c}\right)^{2}+\left(\sigma_{x y}^{c}\right)^{2}\right]$ turns out to be only a little smaller, $0.98 h / e^{2}$ instead of $\rho_{x x}^{c}=1 h / e^{2}$, for the transition point in the lowest Landau band, but with $0.23 h / e^{2}$ somewhat larger than $\rho_{x x}^{c}=0.2 h / e^{2}$ at the second transition. As a further test of the suggested general influence of the eigenfunction correlations on the critical conductance, we investigated also a two-dimensional disordered system with spin-orbit interactions. Using the Ando model, we obtained from transfer matrix calculations a critical conductance $g_{c}^{s}=$ $(1.43 \pm 0.05) e^{2} / h$ and, via direct diagonalization and a multifractal analysis of the critical eigenfunctions, an information dimension $d^{s}(1)=1.89$. We find again that both quantities are perfectly related by a symplectic version of Eq. [4] [33].

In conclusion, we have shown that in the critical quantum Hall regime both the averaged conductance of square samples and the conductivity of very long strips converge to the same universal value $\simeq 0.6 e^{2} / h$ in the limit of large system size. This value does neither depend on the considered disorder realizations nor on the Landau level index, and it is consistent with theoretical predictions that take in to account multifractal eigenstate correlations at quantum critical points.

PM thanks VEGA, grant No. 2/3108/2003, for financial support and PTB for its hospitality.

[1] H. P. Wei, D. C. Tsui, M. A. Paalanen, and A. M. M. Pruisken, Phys. Rev. Lett. 61, 1294 (1988).

[2] S. Koch, R. J. Haug, K. v. Klitzing, and K. Ploog, Phys. Rev. Lett. 67, 883 (1991).
[3] J. T. Chalker and P. D. Coddington, J. Phys. C: Solid State Phys. 21, 2665 (1988).

[4] B. Huckestein and B. Kramer, Phys. Rev. Lett. 64, 1437 (1990).

[5] Y. Huo and R. N. Bhatt, Phys. Rev. Lett. 68, 1375 (1992).

[6] H. Levine, S. B. Libby, and A. M. M. Pruisken, Phys. Rev. Lett. 51, 1915 (1983). A. M. M. Pruisken, Phys. Rev. B 31, 416 (1985).

[7] M. P. A. Fisher, G. Grinstein, and S. M. Girvin, Phys. Rev. Lett. 64, 587 (1990). M. P. A. Fisher, Phys. Rev. Lett. 65, 923 (1990).

[8] D.-H. Lee, S. Kivelson, and S.-C. Zhang, Phys. Rev. Lett. 68, 2386 (1992).

[9] S. Kivelson, D.-H. Lee, and S.-C. Zhang, Phys. Rev. B 46, 2223 (1992).

[10] Y. Huo, R. E. Hetzel, and R. N. Bhatt, Phys. Rev. Lett. 70, 481 (1993).

[11] L. W. Wong, H. W. Jiang, N. Trivedi, and E. Palm, Phys. Rev. B 51, 18033 (1995).

[12] D. Shahar et. al., Phys. Rev. Lett. 74, 4511 (1995).

[13] J. T. Chalker and G. J. Daniell, Phys. Rev. Lett. 61, 593 (1988).

[14] T. Ando, Phys. Rev. B 40, 9965 (1989).

[15] Z. Wang, B. Jovanović, and D.-H. Lee, Phys. Rev. Lett. 77, 4426 (1996).

[16] S. Cho and M. P. A. Fisher, Phys. Rev. B 55, 1637 (1997).

[17] T. Ohtsuki, K. Slevin, and B. Kramer, Physica E 22, 248 (2004).

[18] X. Wang, Q. Li, and C. M. Soukoulis, Phys. Rev. B 58, 3576 (1998).

[19] D. G. Polyakov, Phys. Rev. Lett. 81, 4696 (1998).

[20] T. Koschny and L. Schweitzer, Phys. Rev. B 67, 195307 (2003).

[21] T. Koschny, H. Potempa, and L. Schweitzer, Phys. Rev. Lett. 86, 3863 (2001).

[22] A. MacKinnon, Z. Phys. B 59, 385 (1985). L. Schweitzer, B. Kramer, and A. MacKinnon, Z. Phys. B 59, 379 (1985).

[23] J. B. Pendry, A. MacKinnon, and P. J. Roberts, Proc. R. Soc. Lond. A 437, 67 (1992).

[24] D.-H. Lee, Z. Wang, and S. Kivelson, Phys. Rev. Lett. 70, 4130 (1993).

[25] H. Aoki, Phys. Rev. B 33, 7310 (1986).

[26] B. Huckestein and L. Schweitzer, in Proceedings of the International Conference, Würzburg 1990, edited by G. Landwehr (Springer Series in Solid-State Sciences 101, Springer, Berlin, 1992), pp. 84-88.

[27] W. Pook and M. Janßen, Z. Phys. B 82, 295 (1991).

[28] B. Huckestein, B. Kramer, and L. Schweitzer, Surface Science 263, 125 (1992).

[29] B. Huckestein and L. Schweitzer, Phys. Rev. Lett. 72, 713 (1994).

[30] J. T. Chalker, Physica A 167, 253 (1990).

[31] T. Brandes, B. Huckestein, and L. Schweitzer, Annalen der Physik 5, 633 (1996).

[32] V. I. Fal'ko and K. B. Efetov, Europhysics Letters 32, 627 (1995).

[33] V. I. Fal'ko and K. B. Efetov, Phys. Rev. B 55, 17413 (1995).

[34] F. Evers, A. Mildenberger, and A. D. Mirlin, Phys. Rev. B 64, 241303 (2001).

[35] R. Klesse and M. Metzler, Europhys. Lett. p. 229 (1995).

[36] R. Klesse and M. Metzler, Int. J. Mod. Phys. C 10, 577 (1999).

[37] T. Terao, T. Nakayama, and H. Aoki, Phys. Rev. B 54, 10350 (1996). T. Terao, Phys. Rev. B 56, 975 (1997).

[38] B. Jovanović and Z. Wang, Phys. Rev. Lett. 81, 2767 (1998).

[39] M. Janssen, M. Metzler, and M. R. Zirnbauer, Phys. Rev. B 59, 15836 (1999).

[40] P. Cain, R. A. Römer, M. Schreiber, and M. E. Raikh, Phys. Rev. B 64, 235326 (2001). 